\title{
Characterization of the serological biomarkers associated with Sjögren's syndrome in patients with recalcitrant dry eye disease
}

\section{Cynthia Matossian ${ }^{1,2}$ Joan Micucci'}

'Matossian Eye Associates, Doylestown, PA, USA; ${ }^{2}$ Department of Ophthalmology, Temple University School of Medicine, Philadelphia, PA, USA

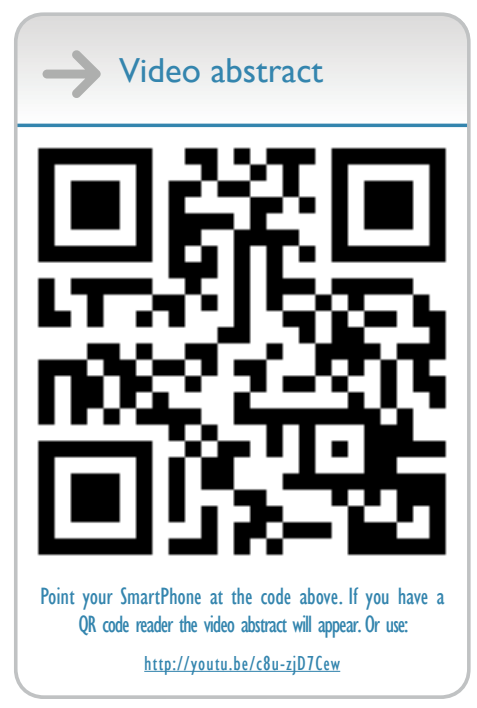

Correspondence: Cynthia Matossian Matossian Eye Associates, 50I Hyde Park, Doylestown, PA 18902, USA

$\mathrm{Tel}+\mathrm{I} 2152309200$

Fax + I 2152309292

Email cmatossian@matossianeye.com
This article was published in the following Dove Press journal:

Clinical Ophthalmology

20 July 2016

Number of times this article has been viewed

Purpose: The purpose was to characterize the biomarkers associated with Sjögren's syndrome (SS) identified in the serological samples of patients with recalcitrant dry eye disease; additionally, the modalities utilized in the treatment of dry eye disease were evaluated for subsets of patients with and without SS.

Patients and methods: Data for this retrospective, single-center, pilot study were based on a chart review of 48 sequential patients with recalcitrant dry eye who were evaluated for SS via serological analysis. Data presented include the presence of the autoantibodies identified through the serological biomarker analysis and identification of the concurrent dry eye treatment modalities. Results: Eleven out of 48 patients (23\%) tested positive for biomarkers associated with SS. Autoantibodies for salivary protein-1, parotid secretory protein 1, and carbonic anhydrase VI, markers associated with the early development of SS, were detected in $91 \%$ (ten out of eleven) of the patients who tested positive for SS, whereas $27 \%$ (three out of eleven) of patients tested positive for the traditional SS markers, SS-A and/or SS-B. Common treatment modalities utilized in SS patients included omega-3 supplements (82\%), topical cyclosporine (74\%), and artificial tear solutions (64\%), as compared to omega-3 supplements $(80 \%)$, hot-mask therapy $(77 \%)$, and artificial tear solutions (77\%), in SS-negative patients.

Conclusion: Evaluation for salivary protein-1, parotid secretory protein 1, and carbonic anhydrase VI biomarkers allows for identification of a subset of patients with biomarkers associated with SS that may not be identified through the traditional assessments (SS-A/SS-B). Earlier recognition of SS biomarkers allows for a confirmatory diagnosis and appropriate management of this systemic, progressive condition.

Keywords: Sjögren's syndrome, dry eye, autoantibodies, autoimmune

\section{Introduction}

Dry eye disease (DED), also known as keratoconjunctivitis sicca, is a complex condition that affects the ocular surface. Alterations in the quality and/or quantity of the tear film result in an instable tear film. Patients with DED report ocular discomfort and irritation in conjunction with photophobia and blurred/fluctuating vision. ${ }^{1}$ Multiple etiologies may contribute to the various manifestations of DED, with inflammation generally playing a central role in the pathophysiology of the condition. ${ }^{1,2}$

Classification systems have been developed to categorize the range of subtypes of DED, primarily emphasizing the causative factors of the disease as well as the nature of the affected components of the lacrimal functional unit. ${ }^{1,3-5}$ The primary subtypes of DED recognized by the classification system presented in the International Dry Eye WorkShop (2007) include aqueous-deficient dry eye and evaporative dry eye. 
The category of aqueous-deficient dry eye is divided into two main subtypes of Sjögren's syndrome (SS) and nonSjögren's syndrome DED. ${ }^{1}$

$\mathrm{SS}$ is a systemic autoimmune condition that affects the exocrine glands. SS is a progressive condition that often affects the salivary glands, resulting in symptoms of dry mouth, and the lacrimal glands, causing a form of DED. The exact triggers or causative factors involved in the development of SS are unknown; however, as a progressive autoimmune condition, chronic stimulation of the immune system likely plays a central role in the pathophysiology. ${ }^{6,7}$

The diagnosis of a patient with SS often occurs years after the initial onset of symptoms, such as dry mouth and the ocular irritation/discomfort associated with DED, with the average time frame for diagnosis ranging from 3.9 to 10.4 years. $^{8,9}$ In addition, the detection of autoantibody biomarkers via serological testing is generally required for a confirmatory diagnosis of SS. ${ }^{10}$ Traditionally, the markers for SS have consisted of anti-SS-A (Ro) or SS-B (La) antibodies for primary SS, elevated antinuclear antibodies (ANA), or the presence of rheumatoid factor (RF), indicating a diagnosis of SS secondary to another autoimmune condition. Recently, a set of novel autoantibodies has been identified that appears to be associated with the early stages of SS. Detection of autoantibodies to salivary protein-1 (SP-1), parotid secretory protein 1 (PSP-1), and carbonic anhydrase VI (CA-6) offers the opportunity to identify the development of an autoimmune condition that may have been missed by screening for only the traditional SS biomarkers. ${ }^{11,12}$

The diagnosis and treatment of patients with DED can be challenging due to the complex etiology of the condition and a frequent lack of correlation between the signs and symptoms of DED. Early identification of DED patients with SS aids in obtaining a confirmatory diagnosis and management of patients with directed treatment in a timely manner. The purpose of the retrospective pilot study was to characterize the biomarkers associated with SS identified in the serological samples of patients with recalcitrant DED; additionally, the modalities utilized in the treatment of DED were evaluated for subsets of patients with and without serum markers associated with SS.

\section{Materials and methods}

This retrospective clinical pilot study was conducted at a single-center, multispecialty ophthalmology practice. All assessments and patient queries were conducted at the clinical site by certified investigators and technicians. Study documents, including the patient data collection file, were submitted to the Sterling Institutional Review Board
(Atlanta, GA, USA) for review; the investigator was granted an exemption from full review, including approval of a waiver of authorization for the use/disclosure of protected health information based on the nature of the study as a retrospective chart review, and a minimal perceived risk to patients as no identifying information is included or presented.

The patient profiles presented include data from 48 sequential patients tested for biomarkers associated with SS using the Sjö serological diagnostic panel (Bausch \& Lomb, Inc., Bridgewater, NJ, USA) with test services and results interpretation provided by IMMCO Diagnostics, Inc. (Buffalo, NY, USA). Inclusion criteria for the study were based on a demonstration of recalcitrant DED and the ability to provide a blood sample for serological testing. Samples for serological testing were obtained during the assessment of the patient's DED. Patients were included in the study who did not demonstrate the expected improvement in their signs and symptoms of DED with traditional treatment modalities. Patients reporting dry mouth concurrent with dry eye symptoms were also included in the study population. No other criteria were required for inclusion in the study. Exclusion criteria for the study centered on the ability to obtain serological test results to quantify the levels of the biomarkers associated with SS. Patients were excluded from the study population based on a lack of serological test results (test results were not available or patients refused to have the test performed). No other exclusion criteria associated with past/present medical history were utilized.

Patient data were collected from the medical records based on a retrospective chart review. Demographic information (age, sex, and ethnicity), Sjö test results, and concurrent use of the following dry eye treatment modalities were collected from the chart review: artificial tear solutions, artificial tear ointments, microwavable hot mask therapy (one or more times per day), cyclosporine ophthalmic emulsion $0.05 \%$ (Restasis $^{\circledR}$, Allergan, Inc., Irvine, CA, USA), intense pulsedlight treatments, and oral omega- 3 fatty acid (re-esterified triglyceride form) nutritional supplements (Physician Recommended Nutriceuticals, Plymouth Meeting, PA, USA).

Blood samples for serological analysis were collected at the study center through a finger prick with a provided lancet in the serological diagnostic panel kit. Blood samples were collected on the sample card designed for use with the diagnostic panel and submitted to IMMCO Diagnostics for analysis of the serological biomarkers. A study report was returned to the study site for each patient that included measurements for each individual biomarker (PSP-1, SP-1, CA-6, SS-A, SS-B, RF, and ANA) and an interpretation of the results based on the relative levels for each analyte. 
Samples were determined to be positive or negative for each biomarker based on a predetermined threshold titer range, which were noted on the sample report.

No calculations were conducted with respect to the sample size of the study population due to the exploratory nature of this pilot study. Descriptive statistics were prepared for the patient demographic information of age (mean, median, minimum, and maximum), sex (\%), and ethnicity (\%). Patient subgroups were determined based on the results of the serological testing; patients testing positive for biomarkers associated with SS, patients testing negative for biomarkers associated with SS, and patients for whom an insufficient quantity of blood sample (quantity not sufficient [QNS]) was indicated on the diagnostic panel study report. Additionally, the individual serological markers (anti-SS-A, anti-SS-B, ANA, RF, SP-1, PSP-1, and CA-6) were evaluated (\% of patients who tested positive for each serological marker or marker group) for the patient subgroup that tested positive for SS biomarkers. Additionally, usage of dry eye treatment modalities was analyzed (\%) for all patients in the study population and for each patient subgroup.

\section{Results}

Six patients were excluded from the study based on a lack of serological test results due to either the patient's refusal to provide a sample for analysis or that the serological test results were not available for evaluation of the patient's immunological biomarkers. A total of 48 patients met the inclusion criteria for the study and were evaluated in this retrospective study analysis. The demographic information collected from the patients is listed in Table 1 for the overall study population and the patient subgroups based on the results of the serological testing. A numerically higher

Table I Patient demographics

\begin{tabular}{|c|c|c|c|c|}
\hline & $\begin{array}{l}\text { Overall } \\
(\mathrm{N}=48)\end{array}$ & $\begin{array}{l}\text { Positive } \\
(n=I I)\end{array}$ & $\begin{array}{l}\text { Negative } \\
(n=30)\end{array}$ & $\begin{array}{l}\text { QNS } \\
(n=7)\end{array}$ \\
\hline \multicolumn{5}{|l|}{ Age (years) } \\
\hline Mean (SD) & $62(14)$ & 59 (I5) & $62(14)$ & $69(13)$ \\
\hline Median & 64.5 & 61 & 64.5 & 74 \\
\hline Min & 26 & 26 & 26 & 44 \\
\hline Max & 82 & 74 & 81 & 82 \\
\hline \multicolumn{5}{|l|}{ Sex $(\%)$} \\
\hline Male & 17 & 9 & 20 & 14 \\
\hline Female & 83 & 91 & 80 & 86 \\
\hline \multicolumn{5}{|l|}{ Ethnicity (\%) } \\
\hline White & 83 & 64 & 93 & 71 \\
\hline Black & 15 & 27 & 7 & 29 \\
\hline Hispanic & 2 & 9 & 0 & 0 \\
\hline
\end{tabular}

Note: $\mathrm{N}=48$.

Abbreviations: Min, minimum; Max, maximum; QNS, quantity not sufficient; $\mathrm{SD}$, standard deviation. percentage of female patients was observed in the subgroup that tested positive for serological biomarkers associated with SS (91\% vs $83 \%$ in the overall study population; Table 1).

Based on the results of the serological testing for biomarkers of SS, $23 \%$ (eleven out of 48 ) of patients tested positive for biomarkers associated with SS, 62\% (30 out of 48) of patients tested negative for biomarkers associated with SS, and the results for $15 \%$ (seven out of 48 ) of patients were returned designated QNS, and were thus unable to be evaluated. The frequency of detection for specific biomarkers is portrayed in Figure 1. Novel biomarkers (SP-1, PSP-1, and CA-6) associated with the early development of SS were detected in $91 \%$ (ten out of eleven) of the patients who tested positive for SS biomarkers, whereas 27\% (three out of eleven) of patients tested positive for the traditional SS markers, SS-A and/or SS-B. ANA and RF were each detected in $18 \%$ (two out of eleven) of the patients testing positive for biomarkers associated with SS.

Treatment options that were utilized by patients in the overall study population, patients testing positive for $\mathrm{SS}$ biomarkers, and patients testing negative for SS biomarkers are displayed in Figure $2 \mathrm{~A}-\mathrm{C}$, respectively (treatment modalities utilized by the seven patients for which a QNS result was returned in the diagnostic study report are not included in the summary statistics presented in Figure 2). In the overall study population, nutritional supplements and artificial tear solutions were reported as the most frequently utilized treatment modalities, followed by hot mask therapy and topical cyclosporine (Figure 2A). In patients who tested positive for SS biomarkers oral nutritional supplements, topical cyclosporine, and artificial tear unguents were observed to have higher frequency of usage as compared to the patients

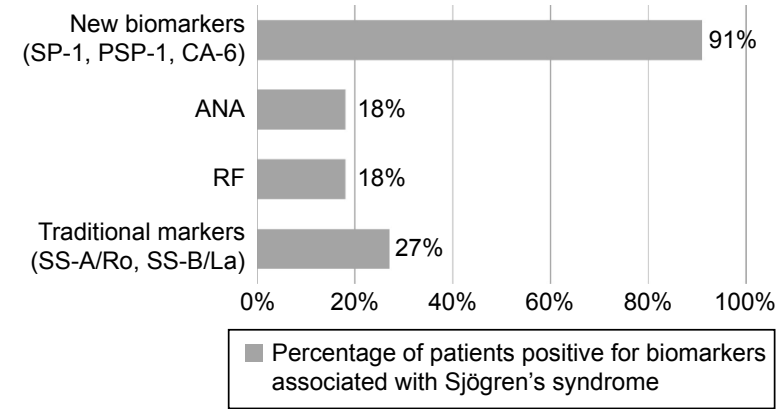

Figure I Distribution of the biomarkers associated with Sjögren's syndrome based on test results.

Notes: The percentages of patients who tested positive for individual biomarker subsets is depicted. The biomarker subsets include the new biomarkers, SP-I, PSP-I, and CA-6; ANA; RF; and the traditional biomarkers for Sjögren's syndrome, SS-A/Ro and SS-B/La. N=II.

Abbreviations: ANA, antinuclear antibodies; CA-6, carbonic anhydrase VI; PSP-I, parotid secretory protein I; SP-I, salivary protein-I; RF, rheumatoid factor. 

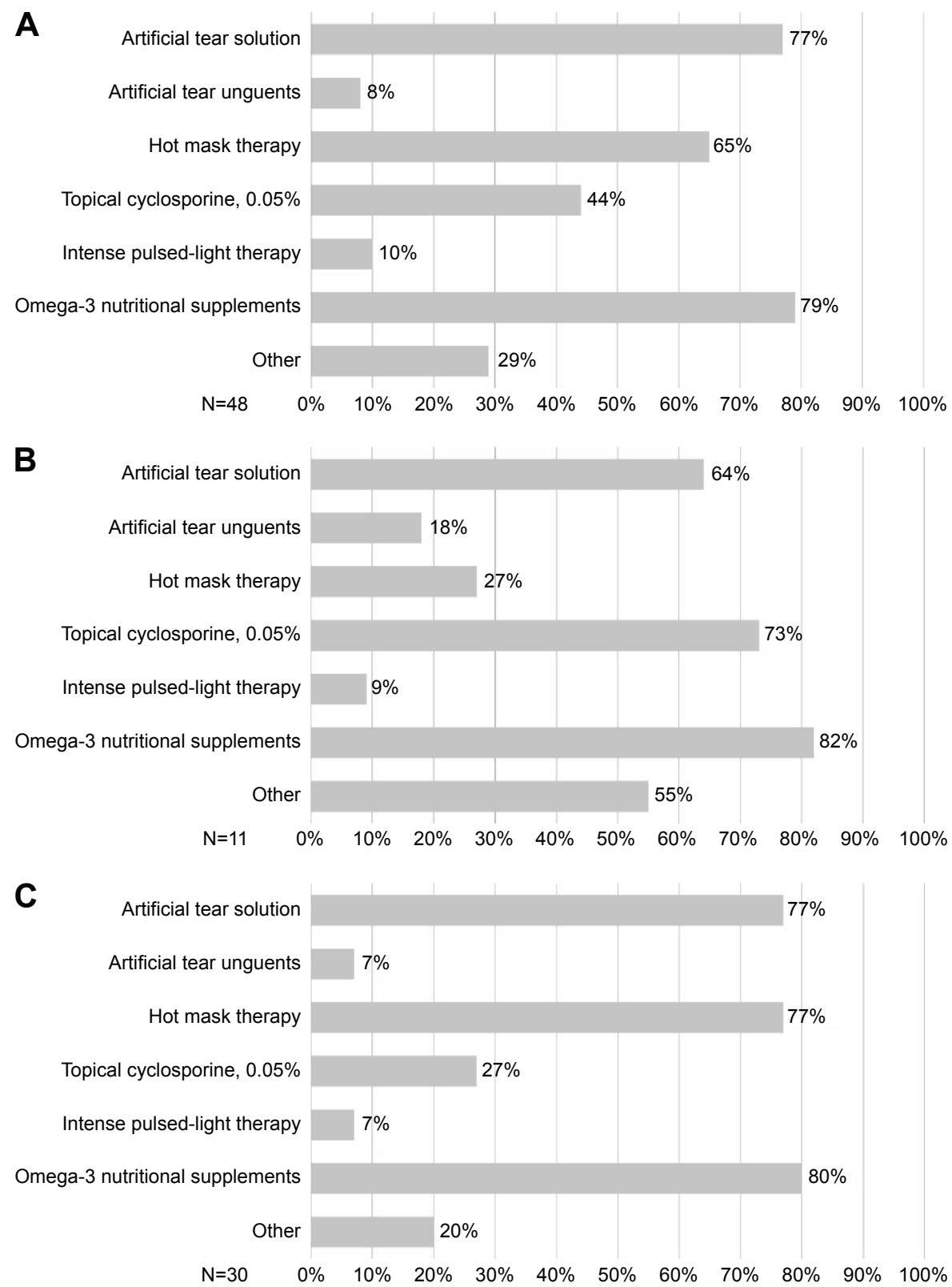

Percentage of total patients

Figure 2 Treatment options utilized by patients in the study population.

Notes: The treatment options for dry eye disease reported as utilized by patients in the study population are depicted as the percentages reported for the total population (A), patients who tested positive for biomarkers associated with Sjögren's syndrome (B), and patients who tested negative for biomarkers associated with Sjögren's syndrome (C). The treatment options reported by patients include: artificial tear solutions, artificial tear unguents, hot mask therapy, topical cyclosporine, $0.05 \%$, intense pulsed light therapy, omega-3 nutritional supplements, and "other" treatment options.

who tested negative for serological biomarkers or the overall study population (Figure 2B). A higher percentage of patients who tested negative for SS biomarkers reported use of hot mask therapy as compared to the overall population and SS positive patients while the lowest frequency of topical cyclosporine usage was recorded in this subgroup (Figure 2C).

A higher number of patient samples were returned as QNS during the early phase of utilizing the serological diagnostic test (February-July 2014) versus the later stage of patient sample collection (after July 2014). Six out of seven patients whose serological samples were returned as QNS were submitted in the initial portion of the study.

\section{Discussion}

The primary purpose of this study was to characterize the serological biomarkers associated with SS identified 
in patients with recalcitrant DED. The serological profile of the patients who were determined to be positive for SS biomarkers indicated that nearly all of this subset of patients expressed autoantibodies for the recently identified biomarkers associated with the early development of SS: SP-1, PSP-1, and CA-6. These novel biomarkers (SP-1, PSP-1, and CA-6) associated with the early development of SS were detected in $91 \%$ (ten out of eleven) of the patients who tested positive for SS biomarkers, whereas $27 \%$ (three out of eleven) of patients tested positive for the traditional SS biomarkers, SS-A and/or SS-B. Therefore, testing patients with recalcitrant DED with these new biomarkers may aid in the earlier diagnosis of SS. The novel biomarkers were also detected in the serological samples of the three patients who tested positive for the classical SS markers (SS-A and/or SS-B), indicating that the autoantibodies to SP-1, PSP-1, and CA- 6 apparently continue to be expressed beyond the early stages of SS.

The patient population in this study included 48 sequential subjects with recalcitrant DED. Failure to achieve improvement in the clinical signs of dry eye and reported symptoms by the subjects, despite conventional therapy, were the primary criteria used to select patients for serological testing and inclusion in this pilot study. Although a more selective set of inclusion and exclusion criteria may be necessary for a multicenter study with a larger patient population, the Investigator determined that the reported inclusion criteria were sufficient to warrant serological testing based on clinical experience. Despite the relatively small size of the patient population in this single-center study, $23 \%$ of the patients tested positive for biomarkers associated with SS. A majority of the overall patient population was female (83\%), and the preponderance of female patients was increased in the subgroup of patients who tested positive for SS (91\%). The high ratio of female to male patients identified with SS biomarkers in the present study agrees with the historically reported epidemiological ratio of 9:1 female to male ratio; ${ }^{13}$ however, the high starting percentage of female subjects in the present study population limits the ability to draw conclusions based on sex distribution of patients testing positive for SS biomarkers.

The assessment of the patient profile of treatment modalities for DED revealed that the use of omega-3 nutritional supplements, artificial tears, and hot mask therapy were the most commonly utilized therapies in the overall study population (Figure 2A). Interestingly, topical cyclosporine $(0.05 \%)$ was recorded as therapy used by $73 \%$ of the subgroup of patients who tested positive for SS biomarkers, as compared to only $27 \%$ of patients who tested negative based on serological markers. In contrast, a higher percentage of patients with a negative biomarker test result reported the use of hot mask therapy (77\%) in comparison to only $27 \%$ of patients who tested positive for SS biomarkers. The inverse profile of these treatment modalities may offer insight into patients who were more likely to present with a more prominent aqueous-deficient component of DED as compared to meibomian gland dysfunction. The limited size of the study population in this pilot investigation limits the ability to draw broad conclusions based on the profile of treatment modalities for the subgroups identified in this study; additional clinical evaluation would be required to confirm this hypothesis.

Samples from a total of seven patients were returned as QNS regarding the serological analysis. The high rate of samples returned as QNS during the early phase of the study (February-July, 2014) is attributed to the general inexperience of the clinic staff in obtaining the blood samples to be sent for analysis. Once the clinic staff had gained experience in collecting the samples, only one QNS result was returned in the latter half of the study period. The relatively high frequency of QNS results in the early phase highlights the necessity for consistent staff training and strict adherence to the sample collection procedure. Recent modifications in the design of the lancets used to collect the blood samples for analysis and the option to order sample collection via venipuncture for serological analysis from a centralized laboratory may also serve to reduce the frequency of QNS results of the diagnostic panel testing.

The conduct of this retrospective pilot study at a singlecenter is a limitation of this study. Expansion of the number of study centers and total patient population would enhance the ability to extrapolate the study findings, particularly in regard to the characteristic profile of patients with and without the biomarkers associated with SS. Despite the heightened awareness of SS by clinicians and the availability of a centralized serological diagnostic testing, the time lag for the diagnosis of SS remains at several years following the onset of patient symptoms. ${ }^{8,9}$

\section{Conclusion}

As an autoimmune disease, $\mathrm{SS}$ is considered a relatively rare condition. In the present study population, $23 \%$ of the patients tested positive for serological biomarkers associated with SS, highlighting the potential that SS may be more common than expected in our patients with recalcitrant DED. Additionally, the observation in this preliminary data set that ten out of 
eleven patients who tested positive had antibodies against the novel biomarkers emphasizes the importance of expanding the scope of general testing for SS to evaluate patients for these early immunological indicators in order to avoid misdiagnosis. Follow-up evaluation of patients identified as positive for the serological biomarkers associated with SS is an important part of management of their condition. Additional measures include referral to a rheumatologist and/or oral medicine specialist for evaluation and salivary gland biopsy, as warranted, to confirm the diagnosis of SS. Earlier recognition and diagnosis of the signs and symptoms of SS allow for a confirmatory diagnosis and appropriate management of this systemic, progressive condition.

\section{Acknowledgments}

This study was supported by Bausch \& Lomb via an independent and unrestricted research grant. Editorial assistance in preparation of the manuscript was provided by Bridge Over Brook, Inc.

\section{Disclosure}

C Matossian: consultant to Bausch \& Lomb Incorporated. The authors report no other conflicts of interest in this work.

\section{References}

1. Methodologies to diagnose and monitor dry eye disease: report of the diagnostic methodology subcommittee of the International Dry Eye Workshop (2007). Ocul Surf. 2007;5:108-152.
2. Stern ME, Schaumberg CS, Pflugfelder SC. Dry eye as a mucosal autoimmune disease. Int Rev Immunol. 2013;32:19-41.

3. Lemp MA. Report of the national eye institute/industry workshop on clinical trials in dry eyes. CLAO J. 1995;21:221-232.

4. Behrens A, Doyle JJ, Stern L, et al. Dysfunctional Tear Syndrome: A Delphi approach to treatment recommendations. Cornea. 2006;25: 900-907.

5. Tomlinson A, Bron AJ, Korb DR, et al. The International Workshop on Meibomian Gland Dysfunction: report of the diagnosis subcommittee. Invest Ophthalmol Vis Sci. 2011;52:2006-2049.

6. Akpek E, Klimava A, Thorne J, Martin D, Lekhanont K, Ostrovsky A. Evaluation of patients with dry eye for presence of underlying Sjögren syndrome. Cornea. 2009;28:493-497.

7. Tincani A, Andreoli L, Cavazzana I, et al. Novel aspects of Sjögren's syndrome in 2012. BMC Med. 2013;11:93.

8. Sjögren's Syndrome - Diagnosis [Sjögren's Syndrome Foundation web site]; 2014. Available from: http://www.sjogrens.org/home/aboutsjogrens-syndrome/diagnosis. Accessed September 29, 2015.

9. Akpek EK, Matthews P, Hahn S, et al. Ocular and systemic morbidity in a longitudinal cohort of Sjögren's syndrome. Ophthalmology. 2015; 122:56-61.

10. Shiboski SC, Shiboski CH, Criswell LA, et al; Sjögren's International Collaborative Clinical Alliance (SICCA) Research Groups. American College of Rheumatology Classification Criteria for Sjögren's syndrome: A data-driven, expert consensus approach in the SICCA cohort. Arthritis Care Res (Hoboken). 2012;64:475-487.

11. Shen L, Suresh L, Lindemann M, et al. Novel autoantibodies in Sjögren's syndrome. Clin Immunol. 2012;145:251-255.

12. Shen L, Kapsoqeorqou EK, Yu M, et al. Evaluation of salivary gland protein 1 antibodies in patients with primary and secondary Sjogren's syndrome. Clin Immunol. 2014;155:42-46.

13. Mavragani CP, Moutsopoulos HM. The geoepidemiology of Sjögrens syndrome. Autoimmun Rev. 2010;9:A305-A310.
Clinical Ophthalmology

\section{Publish your work in this journal}

Clinical Ophthalmology is an international, peer-reviewed journal covering all subspecialties within ophthalmology. Key topics include: Optometry; Visual science; Pharmacology and drug therapy in eye diseases; Basic Sciences; Primary and Secondary eye care; Patient Safety and Quality of Care Improvements. This journal is indexed on Submit your manuscript here: http://www.dovepress.com/clinical-ophthalmology-journal

\section{Dovepress}

PubMed Central and CAS, and is the official journal of The Society of Clinical Ophthalmology (SCO). The manuscript management system is completely online and includes a very quick and fair peer-review system, which is all easy to use. Visit http://www.dovepress.com/ testimonials.php to read real quotes from published authors. 